\title{
Adherence to informed consent standards in Shiraz hospitals: matrons' perspective
}

\author{
Alireza Mohsenian Sisakht ${ }^{1}$, Najme Karamzade Ziarati ${ }^{1}$, Farideh Kouchak ${ }^{2}$, Mehrdad Askarian $^{3,{ }^{*}}$
}

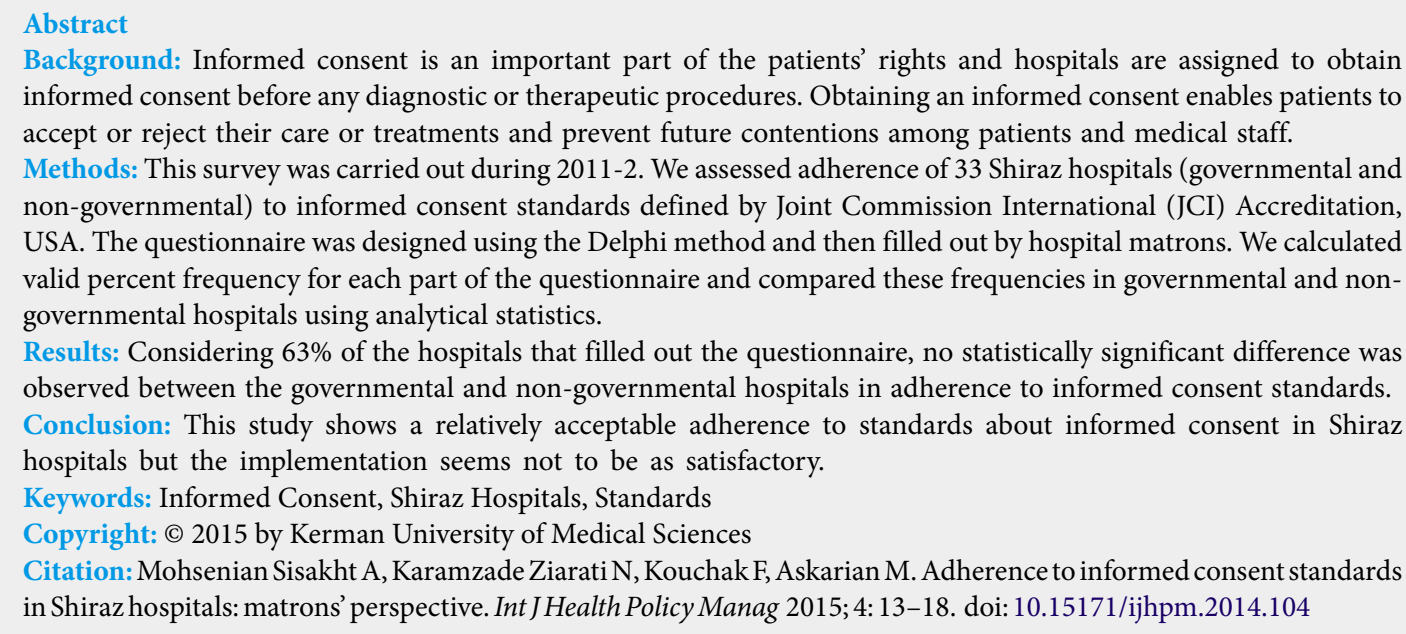
informed consent before any diagnostic or therapeutic procedures. Obtaining an informed consent enables patients to accept or reject their care or treatments and prevent future contentions among patients and medical staff. Methods: This survey was carried out during 2011-2. We assessed adherence of 33 Shiraz hospitals (governmental and non-governmental) to informed consent standards defined by Joint Commission International (JCI) Accreditation, USA. The questionnaire was designed using the Delphi method and then filled out by hospital matrons. We calculated valid percent frequency for each part of the questionnaire and compared these frequencies in governmental and nongovernmental hospitals using analytical statistics.

Results: Considering $63 \%$ of the hospitals that filled out the questionnaire, no statistically significant difference was observed between the governmental and non-governmental hospitals in adherence to informed consent standards. Conclusion: This study shows a relatively acceptable adherence to standards about informed consent in Shiraz hospitals but the implementation seems not to be as satisfactory.

Keywords: Informed Consent, Shiraz Hospitals, Standards

Copyright: ( $\odot 2015$ by Kerman University of Medical Sciences

Citation: Mohsenian Sisakht A, Karamzade Ziarati N, Kouchak F, Askarian M. Adherence to informed consent standards in Shiraz hospitals: matrons' perspective. Int J Health Policy Manag 2015; 4: 13-18. doi: 10.15171/ijhpm.2014.104

Article History:

Received: 4 June 2014

Accepted: 20 October 2014 ePublished: 26 October 2014

*Correspondence to: Mehrdad Askarian Email: askariam@sums.ac.ir

\section{Key Messages}

Implications for policy makers

- Hospital managers in Shiraz should develop measures and protocols in order to enhance their patients' knowledge about "informed consent" standards.

- Policy-makers at the Shiraz University of Medical Sciences should pay more attention to evaluation hospitals about "informed consent" standards, in order to enhance the quality of healthcare services and their patients' satisfaction.

Implications for public

Patients should be aware of their right to take enough information about any decision for their disease, to decide to accept or reject it.

\section{Introduction}

Informed consent, an important part of the patients' rights, is a free and revocable agreement between patients and medical staff about medical processes and involves the nature of procedures including therapeutic or diagnostic ones, risks and benefits and alternative procedures (1). Obtaining an informed consent enables the physician to diagnose the patient's disease while observing his/her rights. In addition, the patient becomes able to accept or reject their offered care or treatments (2). Indeed obtaining informed consent is a way for patient participation in his/her care process (3). An informed consent that is taken in an appropriate way prevents future contentions among patients and medical staff (2). In Iranian patients' bill of rights, two out of 10 items are about informed consent which shows its importance (4). Hospitals are assigned to obtain informed consent before any diagnostic or therapeutic procedures (5).

According to a study in Tehran University of Medical Sciences (TUMS) on measurement of university hospitals' standards defined by the World Health Organization (WHO), the least adherence was found to be in the field of patient and family rights (which included obtaining informed consent) by $47.5 \%$ (6). Another study also conducted in Tehran showed that Iran health ministry standard considers just six options out of the 15 standards defined by the WHO in the field of informed consent. These studies show the need to pay more attention to this important field of medical ethics (7).

Research in other countries has shown that despite the legal coercion to obtain an informed consent in an appropriate way, what actually happens cannot fulfill legal and professional 
requirements and the content of most of them did not meet accepted standards. It seems that systematic education of medical staff is needed to improve the process of obtaining informed consent $(1,8)$.

Taking a signature on the bottom of an informed consent form is not enough to say that this important part of medical ethics is applied in the hospital. Informing the patient about risks, benefits and costs and freedom to accept or reject the procedures is a part of patients' rights and should be considered in the process of obtaining informed consent (9).

Joint Commission International (JCI) defined hospital standards in different issues including patient and family rights. A part of patient and family rights is informed consent which indicates that minimum standards should be considered in hospitals (5). These standards include the route of obtaining informed consent, informing the patients, and defining the procedures which need informed consent and recording (5).

We designed this study to understand the adherence of Shiraz hospitals to these important standards, and to estimate the differences between governmental and non-governmental hospitals, as well as general versus specialized hospitals in Shiraz, from matrons' perspective. In this way, the defects in some parts of informed consent standards become evident and we are able to know whether any defects exist in the some policies, in informed consent taking standards, in defining the procedures that need taking informed consent, in standards of informing patients or in other standards.

\section{Methods}

In this study which was carried out during 2011-2 in Shiraz (with about a million and a half inhabitants), we surveyed 33 hospitals (all of Shiraz hospitals), including governmental, non-governmental, general and specialized ones, to evaluate their adherence to informed consent standards defined by Patient and Family Rights section of JCI "Standards for Hospitals" (5).

The questionnaire had been developed according to those standards determined in the JCI guideline. We translated the questions then after Iranianizing and Islamizing them, we used the Delphi method by participation of 18 specialists including community medicine specialists, residents of community medicine, $\mathrm{PhDs}$ in medical management and both governmental and non-governmental hospital matrons. Considering cultural differences between countries, we adjusted some standards related questions such as standards in blood products transfusion, who can sign the consent form instead the patient, etc. according our culture because of differences from the global view in our Islamic view of blood transfusion and also the persons allowed to give consent instead of the patient. We used the Delphi method to evaluate our questionnaire in different dimensions such as scientific and cultural ones.

According to Skulmoski et al. "the Delphi method is an iterative process used to collect and distill the judgments of experts using a series of questionnaires interspersed with feedback" (10).

The first step of the Delphi method is to design a questionnaire which can be done by literature review, experience and pilot study (10). In this step, as mentioned before, we used the JCI standards after applying some changes to make the questions answerable according to our culture. Then the questionnaire had to be surveyed and accredited by an expert team, comments had to be considered and the new questionnaire given out to them again. Comments of an expert team had to be applied in the final questionnaire (10). After applying the comments by the above-mentioned specialist group, the questionnaire was prepared to be used and we distributed them among the matrons of 33 Shiraz hospitals, and we also explained to them the importance and privacy of their answers face to face.

To assess the reliability of the questionnaire, we calculated Cronbach's alpha in a pilot study $(\alpha=0.909)$, which meant the questionnaire was reliable.

The questions included "clear definition of informed consent process, staff training, obtaining informed consent according the policies, informing patients about their health status, listing procedures and treatments in need of specified consent, collaboration of related physicians and staff in preparing the mentioned list, defining the process for obtaining consent from others (non-patient), justifiability of obtaining consent from others, clarifying the individuals other than the patient who gave consent in the patient's record, informing patient and their family about the scope of a general consent and when it is used by the hospital, defining how a general consent is documented in patients' record, informing patients enough about plan and responsible persons before obtaining consent, informing patients enough about risk and benefits of plan before obtaining consent, informing patients enough about possible alternatives before obtaining consent, informing patients enough about consequences of refusing treatment before obtaining consent, informing patients enough about likelihood of successful treatment before obtaining consent, obtaining consent before surgery, invasive procedure, anesthesia, using blood products and high risk procedures, clarifying the identity of the person who informed patients for above procedures in patients record, documenting the consent in patient record" (5).

The choices of each question were based on 4-pointed options, including: "complete adherence, partial adherence, no adherence, and I do not know". But to calculate the score better we assumed "complete adherence and partial adherence" and also "no adherence and do not know", as equivalent options.

We included all Shiraz hospitals while the exclusion criterion was lack of hospital cooperation. That means 12 hospitals (including governmental and non-governmental and also specialized and general ones) were excluded from this study. In the end, 21 hospitals returned the questionnaires $(63.6 \%$ response rate). Information regarding the hospitals is listed in Table 1.

To increase the validity of the results, we preferred matrons answering the questions, because the hospital matron is a very senior nurse, and he/she has authority and knowledge regarding the hospital policies. We asked them to attach documents about some of the questions, if there were any. Five out of the 21 hospitals $(23.8 \%)$ that participated in the 
Table 1. Characteristics of Shiraz hospitals

\begin{tabular}{|c|c|c|}
\hline Hospital & Type & Specialty \\
\hline Namazi & G & General \\
\hline Shahid Faghihi & G & General \\
\hline Ghotb-e-Din & G & Burns \\
\hline Alavi & NG & General \\
\hline Ordibehesht & NG & General \\
\hline Markazi & NG & General \\
\hline Shahr & NG & General \\
\hline Dena & NG & General \\
\hline Farahmand Far & NG & General \\
\hline Dr. Mir & NG & General \\
\hline Al-Zahra & G & Cardiology \\
\hline Kousar & NG & Cardiology \\
\hline Shooshtari & G & Obstetrics \\
\hline Dastgheib & G & Pediatric \\
\hline Rajaee & G & Trauma \\
\hline Amir & G & Oncology \\
\hline Zeinabie & G & Obstetrics and gynecology \\
\hline Chamran & G & Orthopedic neurosurgery \\
\hline Hafez & G & General \\
\hline Ebn-e-Sina & G & Psychiatry \\
\hline Ali Asghar & G & General \\
\hline
\end{tabular}

$\mathrm{G}=$ governmental; NG= non-governmental

study attached the documents. These documents were about admission sheets, release sheets, and also some procedures such as lumbar puncture, double lumen insertion, etc. Each hospital had its own style in preparing these forms, but their contents were the same in all of hospitals in each form. In each form, the patients identity data was written first, then after explanation of the process, the patients or their care givers were requested to sign the form.

We compared the results of governmental vs. nongovernmental and general vs. specialized hospitals about adherence to informed consent standards.

\section{Statistical analysis}

To understand the adherence to standards, we calculated the percent frequency for each part of the questionnaire and the difference of these frequencies between governmental vs. non-governmental and general vs. specialized hospitals were compared.

\section{Results}

Table 2 shows informed consent standards in rows and adherence to informed consent standards in both governmental and non-governmental hospitals in columns. We also calculated total scores of adherence of governmental and non-governmental hospitals to these standards which are presented in the last row.

Non-governmental hospitals had stricter adherence in almost all aspects of informed consent standards. As we can see in the $9^{\text {th }}$ standard: "Clarifying the individuals, other than the patient, who will give the consent in the patient's record", adherence in both governmental and non-governmental hospitals were $100 \%$, but in the $11^{\text {th }}$ and $17^{\text {th }}$ ( $5^{\text {th }}$ part) standards: "Defining how a general consent is documented in patients' record" and, "Obtaining consent before high risk procedures", governmental hospitals were better.

Another comparison can be made between general and specialized hospitals as presented in Table 3.

In this part, data shows that specialized hospitals have stricter adherence in almost all aspects of informed consent standards. As we can see in the $9^{\text {th }}$ standard: "Clarifying the individuals, other than the patient, who will give the consent in the patient's record", adherence in both specialized and general hospitals were $100 \%$, but in the $1^{\text {st }}, 8^{\text {th }}, 17^{\text {th }}\left(2^{\text {nd }}\right.$ and $4^{\text {th }}$ part) standards, general hospitals were better.

Although the perfect score is a complete adherence (100\%) to all options of the standards, we assumed $>75 \%$ as acceptable adherence, $\leq 75 \%$ and $>25 \%$ as partially acceptable and $<25 \%$ as non-acceptable.

By assuming the above scoring system, $73.9 \%$ of the total of governmental and non-governmental hospitals applied acceptable standards and $26.1 \%$ partially acceptable. In comparison between general and specialized hospitals, the latter performed better and $73.9 \%$ of them applied acceptable standards and $26.1 \%$ partially acceptable. General hospitals were not as good and $43.5 \%$ of them applied acceptable standards and $56.5 \%$ partially acceptable. None of the hospitals was found to be "non-acceptable".

\section{Discussion}

Our data shows a relatively acceptable adherence to informed consent standards in governmental, non-governmental and specialized hospitals but the condition is not as acceptable in general ones. These acceptable results can be affected by the method of collecting data.

The data in this article was obtained from the hospitals' matrons, so poor adherence to obtaining informed consent standards - as shown in other studies in Iran (6,7) - may be hidden. Also this may happen more in non-governmental hospitals than governmental ones. For example, Amini and colleagues' study in Tehran, in which the patients, rather than the matrons, filled out the questionnaires, $31.2 \%$ of patients had complete information about their health status (11). But in our study in which the questionnaires were filled out by hospital matrons, informing patients about their health status was implemented in governmental hospitals by $72.7 \%$ and in non-governmental hospitals by $90 \%$.

The findings of Amini et al. and our study are summarized in Table 4. The Amini et al. study also shows that $11.8 \%$ of patients have information about their treatment plan (11), while in our study, informing patients enough about the plan was applied in governmental hospitals by $78.6 \%$ and in nongovernmental hospitals by $100 \%$.

The patients' awareness about the likelihood of a successful therapeutic plan was $33.3 \%$ in Amini et al.'s study (11) versus $78.6 \%$ and $85.7 \%$ in governmental and non-governmental hospitals in our study.

Patients' information about possible alternatives in Amini et al's study was $21.5 \%$ (12) while informing patients about possible alternatives in our study was $64.3 \%$ in governmental hospitals and $100 \%$ in non-governmental.

As we can see, in all of the above issues, from the patients' viewpoint, it seems, the information they receive was not 
Table 2. Adherence to informed consent standards by governmental and non-governmental hospitals in Shiraz

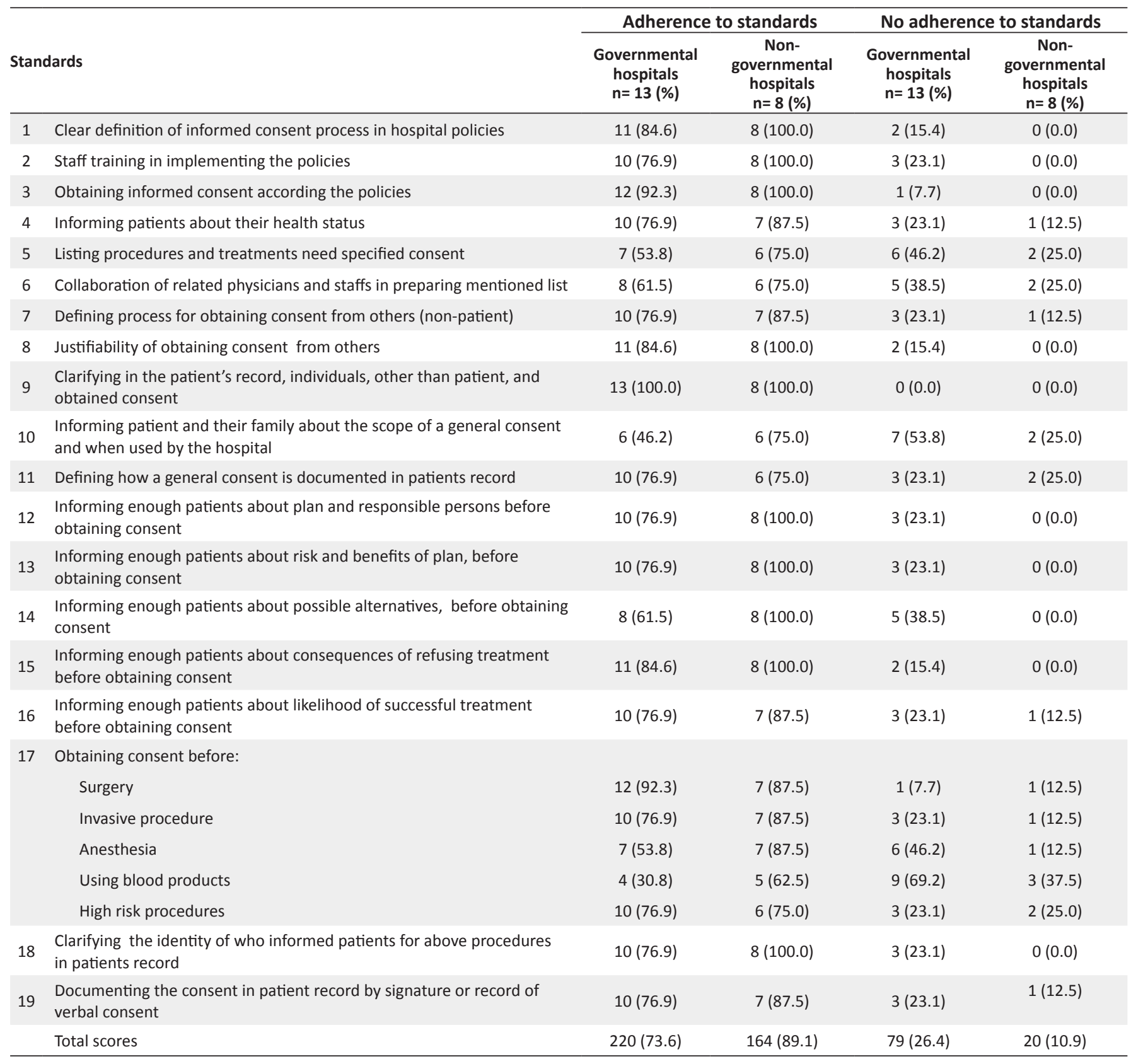

satisfactory but in the matrons' opinions, the information they gave to patients was enough. Also in Sheikhtaheri et al. study in Kashan, it seems, the patients were not satisfied with the information they received before their operations (12). Presumably, these support the idea of underestimating the problems of informed consent standards.

Another assumption is that the data which medical staff think should be given to a patient differ from what the patient wants to hear, so we can see a gap between what is satisfactory for obtaining an informed consent according to medical staff and what is satisfactory for giving an informed consent according to a patient.

It is noteworthy that informed consent is an important issue that addressing this issue from a different perspective such as matrons, patients, clinicians, etc. gives different results. For example, Ogundiran and colleagues' study in Nigeria has surveyed surgeons' opinion around informed consent standards and their results shows that surgeons in Nigeria have enough knowledge about this important issue but their adherence to these standards in practice is lesser than their knowledge (13). For another example we can look the Taylor and Kelner' study; they have studied physicians' perspective about the informed consent regulations. They have surveyed 170 oncologists from eight countries. This study shows that physicians regarded informed consent regulations, a way for decrease effective doctor-patient communication and they also assumed the informed consent regulation as having a negative impact on their patient care (14).

Another point revealed in both Tables 2 and 3 is that the score for application of the determined standards in hospitals is less than their existence. That means despite relatively appropriate determination of (i.e. adherence to) informed consent in 
Table 3. Adherence to informed consent standards by general and specialized hospitals in Shiraz

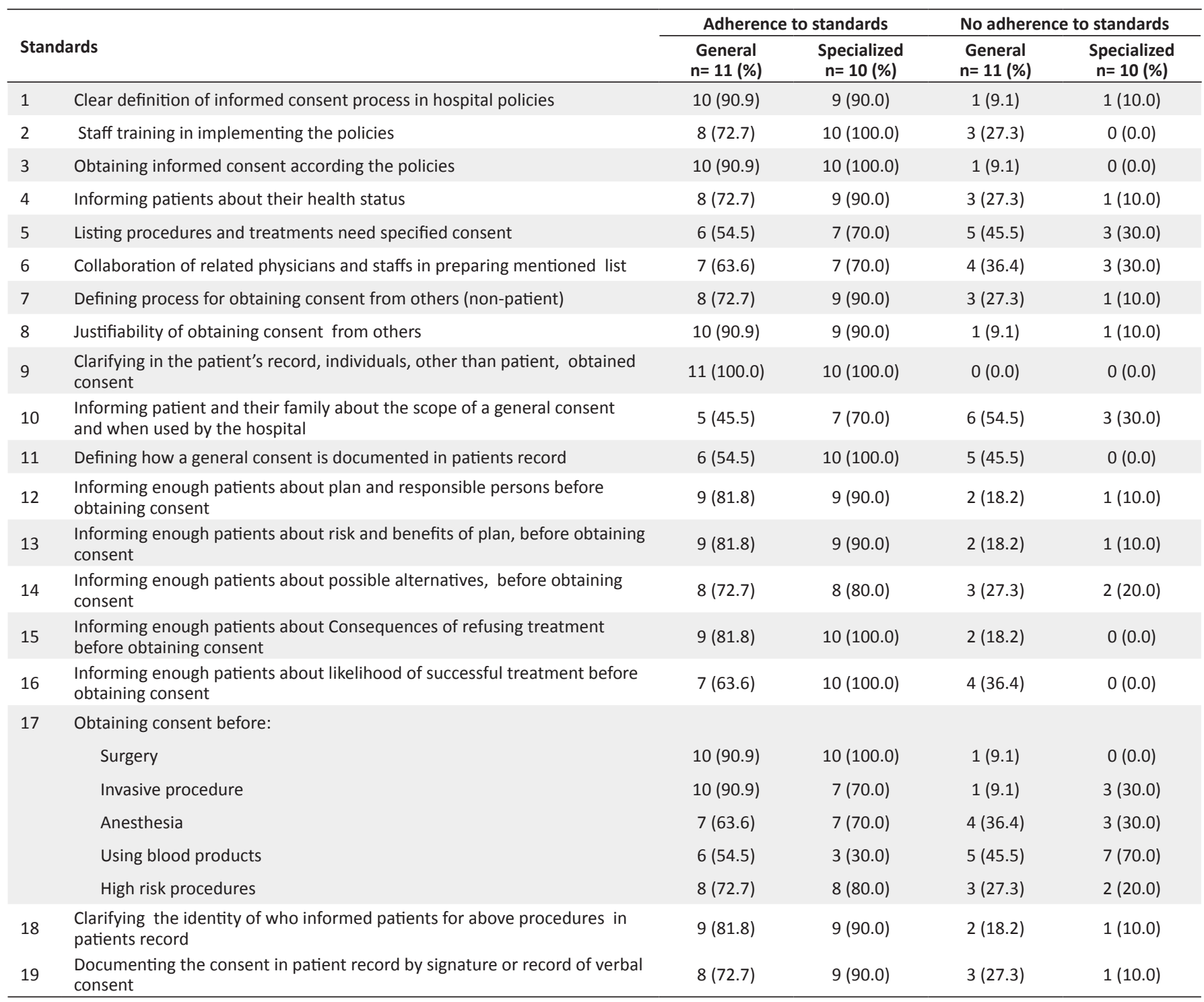

Table 4. Comparison of the same issues from two viewpoints: patients and matrons

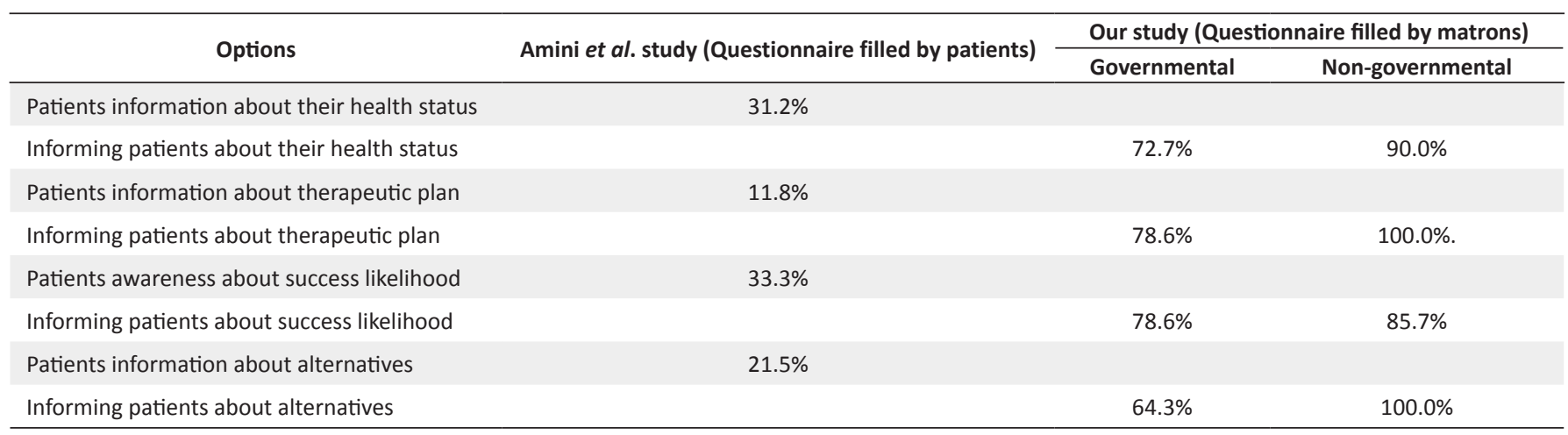

hospital policies, implementation is not satisfactory.

\section{Conclusion}

This study shows a relatively acceptable adherence to standards about informed consent in Shiraz hospitals but according the other studies in Iran, the standards are not applied in a correct way in hospitals and patients are not satisfied. Also, in the present study, the rate of implementing standards is low. That means, despite relatively appropriate determination of informed consent in hospital policies, implementation is not satisfactory.

To upgrade the standards, collaboration of a team including those who determine the policies, talk with patients and inform them is needed. 


\section{Ethical issues}

The study was approved by the ethic committee of Shiraz University of Medical Sciences (SUMS)

\section{Competing interests}

The authors declare that they have no competing interests.

\section{Authors' contributions}

Authors contributed to the publication of this article as follows: study concept and design (AMS, NKZ, FK, and MA); analysis and interpretation of data (AMS, $\mathrm{NKZ}$, and FK ); drafting of manuscript (AMS and NKZ); critical revision of the manuscript (AMS, NKZ, FK, and MA).

\section{Authors' affiliations}

'Student Research Committee, Shiraz University of Medical Sciences, Shiraz, Iran. ${ }^{2}$ Department of Community Medicine, Golestan University of Medical Sciences, Gorgan, Iran. ${ }^{3}$ Department of Community Medicine, Shiraz Nephrourology Research Center, Shiraz University of Medical Sciences, Shiraz, Iran.

\section{References}

1. Bottrell MM, Alpert H, Fischbach RL, Emanuel LL. Hospital Informed Consent for Procedures Forms. Arch Surg 2000; 135; 26-33.

2. Guide for writing a Research Protocol for research involving human participation [Internet]. Available: http://www.who.int/rpc/ research_ethics/guide_rp/en/index.html

3. Hall JK, Boswell MV. Ethics, Law, and Pain Management as a Patient Right. Pain Physician 2009; 12; 499-506.

4. Iranian Center for Research of Medical Ethics and History [homepage on the Internet]. Iranian Patient Rights 2004. Available From: http://mehr.tums.ac.ir/Default.aspx?lang=en

5. Joint Commission International (JCl). Joint Commission International Accreditation Standards for Hospitals. 3rd edition. Oak Brook: JCl; 2008. p. 71-93.
6. Khodayari R, Tourani S, Qaderi A, Salehi M, Jafari H. [Capabilities assessing of teaching hospitals in Iran University of medical sciences in attracting medical tourists according to $\mathrm{JCl}$ patient-oriented standards]. Journal of Hospital 2011; 9: 51-6. [In Persian]

7. Ahmadi M, Khoshgam M, Mohammadpoor A. [Comparative study of the Ministry of Health standards for hospitals with Joint Commission International hospital accreditation standards]. Hakim Research Journal 2007; 10: 45-52. [In Persian]

8. Jukic M, Kvolik S, Kardum G, Kozina S, TomicJuraga A. Knowledge and practices of obtaining informed consent for medical procedures among specialist physicians: questionnaire study in 6 Croatian hospitals. Croat Med J 2009; 50: 567-74.

9. Mirbagher Ajorpaz N, Heidari S, Ranjbar N, Afshar M. Assessment Rate of Being Conscious and Quality of Informed Consent Process in inpatients Undergoing Surgery in Selected Hospitals of Isfahan in 2009. Qom University of Medical Sciences Journal 2011; 3: 53-60. [In Persian]

10. Skulmoski GJ, Hartman FT, Krahn J. The Delphi Method for Graduate Research. Journal of Information Technology Education 2007; 6: 1-21.

11. Amini M, Moosavi S, Mohammadnejad S. The informatory of the inpatients' informed consent: a survey in selected hospitals. Iranian Journal of Medical Ethics and History of Medicine 2009; 2: 61-70.

12. Sheikhtaheri A, Farzandipour M. [Quality of Informed Consent Process and Factors Affecting it among Patients Undergoing Surgery, an Empirical Study in Hospitals of Kashan, Iran]. Hakim Research Journal 2009; 10: 33-41. [In Persian]

13. Ogundrin TO, Adebamowo CA. Surgeons' opinions and practice of informed consent in Nigeria. J Med Ethics 2010; 36: 741-5. doi: 10.1136/jme.2010.037440

14. Taylor KM, Kelner M. Informed consent: the physicians' perspective. Soc Sci Med 1987; 24: 135-43. doi: 10.1016/02779536(87)90246-2 